\title{
SOME REPRESENTATION THEOREMS FOR PARTIALLY ORDERED SETS
}

\author{
E. S. WOLK
}

1. Introduction. In a recent paper [3], Frink has proposed a definition of ideal in a partially ordered set which seems to be a suitable generalization of the usual concept of ideal in a lattice. The purpose of this paper it to show the fruitfulness of Frink's definition by applying some elementary aspects of the theory of such ideals to obtain two representation theorems for partially ordered sets, the second of which is essentially a generalization of a result of Wallman [5]. Using slightly different methods, we also generalize another theorem of Wallman [5], obtaining the result that the structure of a suitably restricted basis (considered as a partially ordered set) for the closed sets of a compact $T_{1}$-space $X$ determines the space $X$ to within homeomorphisms.

2. Definitions and preliminary lemmas. We assume throughout this paper that $P$ is a partially ordered set with elements 0 and $I$. If $A \subset P$, we let $A^{*}=\{p \in P \mid p \geqq a$ for all $a \in A\}$, and $A^{+}=\{p \in P \mid p \leqq a$ for all $a \in A\}$. We write $A^{+*}$ for the set $\left(A^{+}\right)^{*}$, and $A^{*+}$ for the set $\left(A^{*}\right)^{+}$. For any set $A$ we have $A \subset A^{+*}$ and $A^{+*}=\left(A^{+*}\right)^{+*}$, as well as the corresponding dual propositions.

Definition 1 (Frink). A subset $A$ of $P$ is an ideal if and only if for every finite subset $F$ of $A$, we have $F^{*+} \subset A$. A subset $A$ is a $d u a l$ ideal if and only if for every finite subset $F$ of $A$, we have $F^{+*} \subset A$.

In this paper we shall be concerned only with dual ideals. Clearly, for any dual ideal $A, a \in A$ and $b>a$ imply $b \in A$. We call a dual ideal $A$ proper if and only if $A \neq\{I\}$ and $A \neq P$. A proper dual ideal which is a proper subset of no proper dual ideal is called maximal. It is a consequence of Zorn's Lemma that every proper dual ideal is contained in a maximal dual ideal. For a fixed $p \in P$, the set $\{q \in P \mid q \geqq p\}$ is a dual ideal which we call the principal dual ideal generated by $p$, and which we denote by $J_{p}$.

Definition 2. A subset $H$ of $P$ has the finite intersection property if and only if for every finite subset $F$ of $H$ we have $F^{+} \neq\{0\}$.

Lemma 1. If $H$ is a subset of $P$ with the finite intersection property, then there exists a dual ideal $A$ of $P$ such that $H \subset A \neq P$.

Proof. Let $A$ denote the union of all sets of the form $F^{+*}$, taken

Received by the editors January 5, 1955 and, in revised form, August 20, 1955. 
over all finite subsets $F$ of $H$. It is easily shown that $A$ is a dual ideal. We omit the details.

The intersection of any number of dual ideals is again a dual ideal. Thus the set of all dual ideals of any partially ordered set $P$ forms a complete lattice, with the dual ideal $\{I\}$ as its 0 element and $P$ as its $I$ element.

The following definition is a generalization to partially ordered sets of the "disjunction property" introduced by Wallman [5] for lattices.

Definition 3. $P$ is disjunctive if and only if for each pair of elements $a$ and $b$ of $P$ with $a \ddagger b$, there exists $c \in P$ such that $\{a, c\}+\neq\{0\}$ and $\{b, c\}+=\{0\}$.

Definition 4. A set $\Delta$ of maximal dual ideals of $P$ is called a covering family for $P$ if and only if for each $p \in P$ there exists $M \in \Delta$ such that $p \in M$.

Let $\Delta$ be any covering family for $P$. For each $p \in P$, let us write $\Omega_{p}=\{M \in \Delta \mid p \in M\}$. We define $\Omega_{0}$ as the empty set, which we denote by $\varnothing$. The mapping $p \rightarrow \Omega_{p}$ maps $P$ into the set $2^{\Delta}$ of all subsets of $\Delta$. We consider $2^{\Delta}$ as a partially ordered set with respect to the usual inclusion relation.

Two partially ordered sets $P$ and $P^{\prime}$ will be called isomorphic if and only if there exists a 1:1 mapping $f$ of $P$ onto $P^{\prime}$ such that $f(a) \leqq f(b)$ if and only if $a \leqq b . P$ and $P^{\prime}$ will be called anti-isomorphic if and only if there exists a 1:1 mapping $f$ of $P$ onto $P^{\prime}$ such that $f(a) \geqq f(b)$ if and only if $a \leqq b$. We now have the following important lemma, whose proof will be left to the reader.

Lemma 2. If $P$ is disjunctive and $\Delta$ is any covering family for $P$, then the mapping $p \rightarrow \Omega_{p}$ is an isomorphism of $P$ onto a subset of $2^{\Delta}$.

\section{The first representation theorem.}

Theorem 1. A partially ordered set $P$ with 0 and $I$ is isomorphic to $2^{S}$ for some set $S$ if and only if

(i) $P$ is disjunctive, and

(ii) there exists a covering family $\Delta$ for $P$ such that $\bigcap_{M \in \Sigma} M$ is a principal proper dual ideal for every proper nonempty subset $\Sigma$ of $\Delta$.

Proof. Suppose that $P$ satisfies (i) and (ii). We map $P$ into $2^{\Delta}$ by the mapping $p \rightarrow \Omega_{p}$. We show that for any $\Sigma \subset \Delta$, there exists $q \in P$ such that $\Sigma=\Omega_{q}$. This is clearly true for $\Sigma=\varnothing$ or $\Sigma=\Delta$. If $\Sigma \neq \varnothing, \Delta$, then by hypothesis there exists $q \in P, q \neq 0, I$, such that $J_{q}=\bigcap_{M \in \Sigma} M$. Clearly we have $\Sigma \subset \Omega_{q}$. Assume that there exists $M_{0} \in \Omega_{q}$ with $M_{0} \notin \Sigma$. Let $\Sigma^{\prime}=\Delta-\left\{M_{0}\right\}$. Then there exists $r \in P, r \neq 0, I$, such that $J_{r}=\bigcap_{M \in \Sigma^{\prime}} M$. Since, by Lemma $2, I$ is the only element of $P$ which 
is in every $M \in \Delta$, we must have that $r \notin M_{0}$. Hence $J_{r}=\bigcap_{M \in \Sigma^{\prime}} M$ $\nsubseteq M_{0}$; and since $\Sigma \subset \Sigma^{\prime}$, we have also $J_{q}=\bigcap_{M \in \Sigma} M \nsubseteq M_{0}$. But by assumption $q \in M_{0}$ : a contradiction. Hence $\Sigma=\Omega_{q}$. We omit the proof of the converse.

Corollary 1. A partially ordered set $P$ with 0 and $I$ is isomorphic to $2^{S}$ for some finite set $S$ if and only if

(i) $P$ is disjunctive, and

(ii) the covering family $\mathfrak{T}$ of all maximal dual ideals of $P$ satisfies condition (ii) of Theorem 1.

Proof. Let $P$ satisfy (i) and (ii). We have only to show that $\mathscr{M}$ is finite. But it is known that in any Boolean algebra, every dual ideal $J$ is the intersection of all $M \in \mathscr{N}$ which contain $J$ (see $[1$, p. 161, exercise 4]). Hence every dual ideal of $P$ is principal, and $P$ is finite by exercise 3, p. 161 of [1]. Conversely, if $S$ is finite, then all dual ideals of $2^{s}$ are principal, and condition (ii) follows as in Theorem 1.

As a further corollary of Theorem 1 we may easily obtain a wellknown theorem of Birkhoff and Ward [2] (see also [1, p. 170, Theorem 16]). An element $p \neq 0$ of $P$ is called a point if and only if $q<p$ implies $q=0 . P$ is atomic if and only if $a \in P$ and $a \neq 0$ imply there exists a point $p$ with $p \leqq a$. We then have the following result.

CoRollary 2 (BIRKhoff-WARD). If $L$ is a complete atomic lattice with unique complements, then $L$ is isomorphic to $2^{S}$, where $S$ is the set of all points of $L$.

Proof. Clearly $L$ is disjunctive. We take as our covering family $\Delta$ the set of all maximal dual ideals $M_{p}$ which are generated by points. If $q \in L$, let us denote the complement of $q$ by $q^{\prime}$. It is easily shown, as on p. 170 of [1], that if $p$ and $q$ are distinct points of $L$, then $p \leqq q^{\prime}$. Now let $\Sigma$ be a proper nonempty subset of $\Delta$. There exists a point $q$ with $M_{q} \notin \Sigma$, and hence for all $M_{p} \in \Sigma$ we have $p \leqq q^{\prime} \neq I$. Hence $b=\sup \left\{p \mid M_{p} \in \Sigma\right\} \neq I$. But $J_{b}=\bigcap_{M p \in \Sigma} M_{p}$, which is a principal proper dual ideal. Hence condition (ii) of Theorem 1 is satisfied, and our result follows.

4. Another representation theorem. We first give two more definitions.

Definition 5. A dual ideal $J$ is called reducible if and only if $J$ is the intersection of a set of maximal dual ideals.

Definition 6. A dual ideal $K$ is prime if and only if for any reducible dual ideals $J_{1}$ and $J_{2}$ such that $K \supset J_{1} \cap J_{2}$, we have $K \supset J_{1}$ or $K \supset J_{2}$.

Now let $X$ be a $T_{1}$-space. We say that $B$ is a basis for the closed sets 
of $X$ if and only if $B$ is a collection of closed subsets of $X$ with the property that every closed subset of $X$ is the intersection of a set of elements of $B$. We consider such a basis $B$ as a partially ordered set with respect to the relation of set inclusion, adjoining the empty set to $B$ as its 0 element.

TheOREM 2. A disjunctive partially ordered set $P$ in which each maximal dual ideal is prime is isomorphic to a basis for the closed sets of a compact $T_{1}$-space.

Proof. We introduce a topology on the set TT of all maximal dual ideals of $P$. (This topology is a familiar one on the set of maximal ideals of a commutative ring.) If $\Sigma \subset$ T, we define $C(\Sigma)=\{M \mid M$ $\left.\supset \bigcap_{M \in \Sigma} M\right\}$, and $C(\varnothing)=\varnothing$. Clearly we have (i) $\Sigma \subset C(\Sigma)$, (ii) $C(C(\Sigma))=C(\Sigma)$, and (iii) $\Sigma=\left\{M_{0}\right\}$ implies $C(\Sigma)=\Sigma$. We also assert that (iv) $C\left(\Sigma_{1} \cup \Sigma_{2}\right)=C\left(\Sigma_{1}\right) \cup C\left(\Sigma_{2}\right)$. For let $J_{1}=\bigcap_{M \in \Sigma_{1}} M, J_{2}$ $=\bigcap_{M \in \Sigma_{2}} M$. Then $C\left(\Sigma_{1} \cup \Sigma_{2}\right)=\left\{M \mid M \supset J_{1} \cap J_{2}\right\}$. But $J_{1}$ and $J_{2}$ are reducible: hence $M \in C\left(\Sigma_{1} \cup \Sigma_{2}\right)$ implies $M \supset J_{1}$ or $M \supset J_{2}$, which is equivalent to the statement that $M \in \Sigma_{1} \cup \Sigma_{2}$. Since obviously we also have $C\left(\Sigma_{1}\right) \cup C\left(\Sigma_{2}\right) \subset C\left(\Sigma_{1} \cup \Sigma_{2}\right)$, our assertion is proved.

Conditions (i)-(iv) state that we have defined a $T_{1}$-topology on the set $\mathfrak{T}$. Now for each $p \in P$, let $\Omega_{p}=\{M \in \Re \mid p \in M\}$. The sets $\Omega_{p}$ obviously are closed subsets of $\mathfrak{T}$. By Lemma 2 , the mapping $p \rightarrow \Omega_{p}$ is an isomorphism of $P$ into the set of all subsets of $\mathfrak{T}$. To show that the set of all sets $\Omega_{p}$ is a basis, suppose that $\Sigma$ is a closed subset of $\Re$. Let $J=\bigcap_{M \in \Sigma} M$ : then $M \supset J$ if and only if $M \in \Sigma$. But $\bigcap_{p \in J} \Omega_{p}$ $=\{M \mid M \supset J\}=\Sigma$. Thus the sets $\Omega_{p}$ form a basis for the closed sets of the space $\mathfrak{T}$.

The proof that $\mathscr{T}$ is compact is similar to that of Lemma 7, p. 116 , of $[5]$. We omit the details.

Using similar methods we now deduce a theorem which characterizes the lattice of all closed subsets of a $T_{1}$-space. This theorem is related to results of Ore $[4$, p. 772$]$ and $[1$, p. 173, exercise 1$]$.

THEOREM 3. Let $L$ be a complete, atomic, and disjunctive lattice, and $S$ its set of points. $A$ necessary and sufficient condition that $L$ be isomorphic to the lattice of all closed subsets of a $T_{1}$-space $X$ (where $X$ is in $1: 1$ correspondence with $S$ ) is that $p \in S$ and $p \leqq a \cup b$ imply $p \leqq a$ or $p \leqq b$.

Proof. Let $\Delta$ be the covering family for $L$ consisting of all maximal dual ideals $M_{p}$ generated by points. Suppose that $L$ satisfies the condition given in the statement of our theorem (the necessity of this condition is obvious). Then for any $M_{p} \in \Delta$ and any principal dual 
ideals $J_{a}$ and $J_{b}$ such that $M_{p} \supset J_{a} \cap J_{b}$, we have $M_{p} \supset J_{a}$ or $M_{p} \supset J_{b}$. This fact allows us to topologize the set $\Delta$ with the same topology used in Theorem 2, since it implies that condition (iv) (see proof of Theorem 2) is satisfied. Thus $\Delta$ becomes a $T_{1}$-space. For $q \in L$, the mapping $q \rightarrow \Omega_{q}$ maps $L$ isomorphically into $2^{\Delta}$. The sets $\Omega_{q}$ are obviously closed subsets of $\Delta$. Now if $\Sigma$ is any closed subset of $\Delta$, let $b=\sup \left\{p \in S \mid M_{p} \in \Sigma\right\}$. We have $\Omega_{b}=\Sigma$, and hence our theorem is proved.

\section{An application to point-set topology.}

Definition 7. Let $B$ be a basis for the closed sets of a $T_{1}$-space. We say that $B$ is admissible if and only if for every finite subset $F$ of $B$ with $\bigcap_{p \in F} p \neq \varnothing$, there exists $p_{0} \in B$ such that $p_{0} \subset \bigcap_{p \in F} p$.

Any $T_{1}$-space $X$ possesses an admissible basis, since the set of all closed subsets of $X$ is obviously admissible.

Wallman [5] has proved, in essence, that if $X$ is a compact $T_{1}$-space and $L$ is a basis for the closed sets of $X$ which is a disjunctive lattice, then the lattice structure of $L$ determines the space $X$ to within homeomorphisms. We extend this result in the following way.

THEOREM 4. Let $X$ be a compact $T_{1}$-space and let $B$ be any admissible basis for the closed sets of $X$. Then the structure of $B$, considered as $a$ partially ordered set, determines $X$ to within homeomorphisms.

Proof. Let $R(B)$ be the set of all reducible dual ideals of $B$. We partially order $R(B)$ by set inclusion, adjoining the element $B$ as an $I$ element. It is clear that $R(B)$ thus becomes a complete lattice. Our proof of Theorem 4 will consist of showing that the lattice $R(B)$ is anti-isomorphic to the lattice $\mathfrak{F}(X)$ of all closed subsets of $X$.

For each $x \in X$, let $K_{x}=\{p \in B \mid x \in p\}$. We shall prove that $K_{x}$ is a maximal dual ideal of $B$. Since $B$ is an admissible basis, $K_{x}$ has the finite intersection property; and hence by Lemma 1 there exists a maximal dual ideal $M_{x}$ such that $K_{x} \subset M_{x}$. But any proper dual ideal of $B$ has the finite intersection property and hence by the compactness of $X$ there exists $y \in X$ such that $M_{x} \subset K_{y}$. Our $T_{1}$ hypothesis on $X$ now implies that $x=y$, and hence $K_{x}=M_{x}$. Thus every maximal dual ideal of $B$ is a $K_{x}$ for some $x \in X$.

Now let $R$ be any reducible dual ideal of $B$. Then $R=\bigcap_{x \in S} K_{x}$, where $S$ is a subset of $X$; or equivalently, $R=\{p \in B \mid S \subset p\}$. But since each $p \in B$ is closed, we may write $R=\{p \in B \mid \bar{S} \subset p\}$, where $\bar{S}$ is the closure of $S$. The latter representation of $R$ is clearly unique. Conversely, if $S$ is any closed subset of $X$, the set $R(S)=\{p \in B \mid S \subset p\}$ $=\bigcap_{x \in S} K_{x}$ is a reducible dual ideal of $B$. Hence the correspondence $S \leftrightarrow R(S)$ is an anti-isomorphism of $\mathcal{F}(X)$ onto $\Re(B)$. 
The structure of $B$ as a partially ordered set therefore determines the lattice structure of $F(X)$. But the theorem of Wallman mentioned above states that the lattice $\Im(X)$ determines the space $X$, and hence $B$ determines the space $X$.

\section{REFERENCES}

1. G. Birkhoff, Lattice theory, Amer. Math. Soc. Colloquium Publications, vol. 25, 1948.

2. G. Birkhoff and M. Ward, A characterization of Boolean algebras, Ann. of Math. vol. 40 (1939) pp. 609-610.

3. O. Frink, Ideals in partially ordered sets, Amer. Math. Monthly vol. 61 (1954) pp. 223-234.

4. O. Ore, Some studies on closure relations, Duke Math. J. vol. 10 (1943) pp. 761785.

5. H. Wallman, Lattices and topological spaces, Ann. of Math. vol. 39 (1938) pp. 112-126.

UNIVERSITY OF CONNECTICUT 